carried out. This has demonstrated a group of rheumatoids with fairly severe personality disturbances of long standing.

The advantage of administering the E.P.I. test and then carrying out studies in depth has been that it has demonstrated, quite remarkably in some cases, the basic personality problems which have been present from a very early age and therefore cannot be attributable to the rheumatoid processes. The E.P.I. results, on retesting after a considerable period of time, show a remarkable consistency and it is therefore a very useful tool to be used in the initial assessment of patients.

There has been some evidence that in those patients showing perfonality disturbance, evidence of depressive illness, etc., the frequency of episodes of disabling deterioration has been markedly decreased by treating their emotional condition. This study will be the subject of a fuller report to be published later.-I am, etc.

$$
\begin{aligned}
& \text { Department of Physical } \\
& \text { Medicine, } \\
& \text { Addenbrooke's Hospital, } \\
& \text { Cambridge. }
\end{aligned}
$$

Alan J. Rowe.

\section{Spotting Typhoid}

SIR,-Though your leading article "Spotting Typhoid" has long since passed (13 September, p. 607) perhaps I can add some relpful comments.

I have worked in this hospital in western Uganda for eight months. Typhoid is endemic in the area, accounting for 360 admissions last year. The majority present in the classical manner with severe headache, fever, prostration, abdominal pain, and constipation. But I have also seen cases frequently presenting with the single symptom of tinnitus, dizziness, epistaxis, or diarrhoea. Deafness, epididymo-orchitis, and hoarse voice have been among the more unusual presenting symptoms.

We treat them with chloramphenicol for 14 days and the fever of $101^{\circ}-103^{\circ} \mathrm{F}$ $\left(38.5^{\circ}-39.5^{\circ} \mathrm{C}\right.$.) usually returns to normal within four days. Lack of space often dictates discharge after only seven days in hospital. In perhaps 200 cases treated I have seen only one case of sore throat and a leucopenia which could possibly have been due to bone marrow depression.-I am,etc.,

Kabale Hospital,
Uganda.

\section{S. J. Parker.}

$$
\text { Uganda. }
$$

\section{Heat and the I.U.C.D.}

SIR,-Short-wave diathermy is now being frequently prescribed for the pelvis or sacroiliac joints, and in many physiotherapy departments the older techniques of anterior and posterior electrodes is still being used.

Many women patients are now fitted with an intrauterine device yhich is made of plastic, and a nursing sister has raised the question whether it would be safe to have such therapy in view of the risk that the plastic spiral might lose its shape from the heat. I did a number of experiments using an I.U.C.D. in the vagina which so far shows that the device does not become deformed. However, the question of whether the device is affected at given temperatures now requires closer investigation, with the intrauterine temperature being recorded at the same time.

I wish to draw attention to this possible hazard in the hope that those in charge of physiotherapy departments might be able to continue more detailed experiments on the continued reliability of the device when patients are submitted to short-wave diathermy treatment.-I am, etc.

\section{Manchester Victoria Memorial Jewish Hospital,
Manchester 8 .}

B. SANDLER.

\section{Length of a Lippes Loop}

SIR,-I wish to report an unusual complication associated with the insertion of a Lippes loop.

A presterilized Lippes loop size $\mathrm{D}$ in a 1-unit-sized pack was inserted into a multigravid patient according to the instructionsthat is, the loop was pulled into the inserting tube only within a minute of insertion. Before insertion it had been noted that the loop within the packet appeared to conform to the normal dimensions of the loop size D-that is, $30 \mathrm{~mm}$. long by $30 \mathrm{~mm}$. wide. After hal the loop had been inserted into the patient there was severe pain and a small amount of bleeding so that the loop and inserter were withdrawn. At no time had the insertion of the loop been associated with a sense of giving way, as would possibly occur if the myometrium was penetrated. The patient was hospitalized for 24 hours and given on injection of pentazocine $30 \mathrm{mg}$. intravenously. At the end of 24 hours there was no bleeding and no pain.

Closer investigation of the loop within 5 minutes of withdrawal of the loop from the patient disclosed that it had changed its dimensions. It still conformed to the $30 \mathrm{~mm}$. width but the various angles had opened out and the new length had become $50 \mathrm{~mm}$. Two days later this length had remained at $50 \mathrm{~mm}$.

I would like to suggest that it might well be in future a wise precaution for the loop to be not only drawn into the tube as suggested before insertion but withdrawn into the tube and expelled at least once to see if it takes up its pre-tube length rapidly before use on the patient.-I am, etc.,

\section{Cairns, Queensland, Australia.}

\section{Exacerbations in Chronic Bronchitis}

SIR,-It is disappointing that the careful investigations by Dr. Mary Fisher and her colleagues (25 October, p. 187) throw little fresh light on the causes of exacerbations in chronic bronchitis.

During the past two years a study of acute bronchitis in children has been carried out jointly by one of us (I. G.) in his general practice at Roehampton, London, and by the department of virology of the Brompton Hospital, using methods of bacteriological and virological investigation broadly similar to those described by Dr. Fisher and her colleagues. In addition to the children, a small number of adult chronic bronchitics were studied during acute exacerbations in a pilot trial for a larger study which has now begun this autumn.

The criteria which were used to define an acute $\bar{z}$ exacerbation were similar to those of Dr. Fisher $\bar{D}$ and her colleagues. Isolation of viruses was achieved in 12 of 28 episodes as follows: rhinoviruses ( $H$ and $M$ types), three times; influenza A, twice ; parainfluenza type III, twice; M. $\cong$ pneumoniae, once ; respiratory syncytial virus, $\bar{O}$ once; herpes simplex, three times. Serological $\frac{}{0}$ investigations alone were performed in 12 other $\bar{\omega}$ acute episodes, and a rise in complement-fixing $\vec{D}$ titre was found in three of these episodes $\triangle$ (influenza A, once ; respiratory syncytial virus, 0 once; respiratory syncytial virus and influenza A together, once). Thus, if the significance of $\overrightarrow{0}$ herpes simplex is disregarded, we found evidence of an association with viral or myco- $\vec{\omega}$ plasmal infection in $12(30 \%)$ of the 40 episodes which were investigated.

One reason for the discrepancy between our findings and those of Dr. Fisher and her colleagues may lie in the time interval between the onset of the acute episode and the inocula- : tion of tissue cultures. In our study throat $\infty$ and nasal swabs were taken within 48 hours of the onset of the first symptoms. Some- is times this was difficult to determine, and in $ᄋ$ several of the episodes which were investi- $N$ gated with negative results it is possible that the true time interval was longer than this. Tissue cultures were inoculated within three hours of swabbing. In the investigations of $\frac{\Phi}{3}$ Dr. Fisher and her colleagues it would appear that a considerably longer delay occurred in $\mathbb{1}$ most cases between the first onset of symp- $\vec{\varphi}$ toms and both the taking of specimens and 8 the inoculation of tissue cultures.-We are, etc.,

Department of Virology,

IAN GREGG.

Brompton Hospital,

J. M. INGLIS.

\section{Epilepsy and Folate Deficiency}

SIR,-Dr. John Wilson in his review of the drug treatment of epilepsy in childhood (22 November, p. 475) comments that "it is reasonable to remedy folate depletion as necessary." In the present state of knowledge concerning the effects of drug-induced folate deficiency this is hard to dispute, and we are prompted by his statement to mention a technique which we have been using for folate restoration in these children.

While we would agree with Dr. Wilson that documentary evidence concerning the exacerbation of seizures by folate therapy is equivocal, our own experience using standard dosage of folic acid (5 mg. tablets) has been $\omega$ alarming. This has led us to adopt the practice of low-dosage folate replacement. As of our results have been encouraging we suggest that this approach might be more widely used. This would obviate the need to argue the pros and cons of standard-dose folate therapy in epileptic children.

Every epileptic child in our care who is folate deficient is given $100 \mu \mathrm{g}$. of folic acid daily. Using this dose we have obtained satisfactory folate restoration within about three months in the cases we have treated so far. There have been no untoward sequelae.

For prescribing on an inpatient basis, capsules containing $100 \mu \mathrm{g}$. of folic acid are prepared for us by our chief pharmacist. One

\section{,}

